\title{
Factores de riesgo que determinan la transmisión de la leishmaniasis en el valle Llaucano, Chota-Cajamarca
}

\author{
Víctor Zorrilla ${ }^{1}$, María Agüero ${ }^{2}$, Abraham Cáceres ${ }^{1,3}$, Abelardo Tejada ${ }^{1}$, \\ Juan Ticlla ${ }^{4}$, Rosa Martínez ${ }^{2}$
}

Resumen

Objetivos: Determinar los factores de riesgo asociados con la transmisión de leishmaniasis. Material y Métodos: Se entrevistó 727 personas en 140 viviendas de 8 localidades del valle Llaucano, Chota, Cajamarca. Los factores de riesgo fueron analizados mediante el cálculo del odds ratio, con límites de confianza al 95\%, utilizando el pro-grama EpiInfo 2000. Resultados: Se diagnosticó 206 casos de leishmaniasis, 63 lesiones activas y 143 cicatrices. La leishmaniasis se presenta en todos los grupos de edad y ambos sexos están afectados en proporciones similares, siendo más frecuente en menores de 10 años. El alto porcentaje de lesiones en cara y miembros superiores, sugiere una transmisión domiciliaria de la enfermedad. Los factores de riesgo asociados con la transmisión de la leishmaniasis en el valle de Llaucano, son los siguientes: viviendas dispersas ubicadas en áreas rurales (OR 3,97, IC95\% 1,94-8,14), dormir en viviendas improvisadas en medio de las chacras en condiciones precarias (OR 4,59, IC95\% 3,22-6,54), cultivos de café (OR 7,83, IC95\% 3,57-17,17), caña de azúcar (OR 4,99, IC95\% 2,42-10,27) y frutas (OR 3,62, IC95\% 1,72-7,61) en peridomicilio; hacinamiento con 6 ó más personas por vivienda $(O R=3,25$, IC95\% 1,50-7,10); animales domésticos en el domicilio, principalmente perros (OR=4,03, IC95\% 1,98-8,20); flebotominos en el ambiente domiciliario (OR =5,62, IC95\% 2,68-11,82), que ingresan a través de las paredes de "tapial". Paccha Baja, Chontabamba y Matibamba son los focos de más alta transmisión de leishmaniasis. La prevalencia acumulada fue de 283,4/ 1000 hab. Conclusiones: El valle de Llaucano es zona endémica con elevada velocidad de propagación de la leishmaniasis; la transmisión de la enfermedad está asociada a factores de vivienda y comportamiento humano.

Palabras clave Leishmaniasis; factores de riesgo; zonas rurales; áreas de pobreza; Perú, Cajamarca, valle Llaucano.

\section{Risk factors that determine leishmaniasis transmission at Llaucano valley, Chota-Cajamarca}

\section{Abstract}

Objectives: To determine the epidemiology of cutaneous leishmaniasis. Material and Methods: Personal interviews were done to 727 inhabitants of 140 houses in eight localities of Llaucano valley, Chota province, Cajamarca, searching for leishmaniasis cases. The risk factors were analyzed using odds ratio calculation, 95\% confidence interval in each selected variable and Epi Info 2000

\footnotetext{
1 Instituto de Medicina Tropical "Daniel A. Carrión"-UNMSM. Lima, Perú.

2 Facultad de Ciencias Biológicas, UNMSM. Lima, Perú.

3 Instituto Nacional de Salud. Lima, Perú.

4 Dirección Regional de Salud Chota. Cajamarca, Perú.
}

program. Results: Two hundred and six cutaneous leishmaniasis cases were diagnosed, 63 with active lesions and 143 with scars. Leishmaniasis was present in all age groups without sex differences, but children under 10 years old were more affected. High percentage of cases showed lesions in face and arms suggesting intra and peridomiciliary transmission at Llaucano valley. The risk factors for leishmaniasis transmission were identified as following: To have the house located in the rural area $(O R=3,97, C I 95 \% 1,94-8,14)$, to sleep in country shelters $(O R=4,59, C I 95 \% 3,22-6,54)$, to cultivate coffee $(\mathrm{OR}=7,83$, CI95\% 3,57-17,17), fruits (OR=3,62, CI95\% $1,72-7,61)$ and sugar cane (OR=4,99, CI95\% 2,42-10,27), the crowding conditions of life in some families (6 or more persons for each house) $(O R=3,25, C I 95 \% 1,50-7,10)$, the presence of domestic animals around the house, mainly 
dogs (OR=5,62, CI95\% 2,68-11,82), presence of sandflies in the domiciliary environment $(\mathrm{OR}=5,62, \mathrm{CI95 \%} 2,68$ 11,82) that penetrate the "tapia" wall holes. Paccha Baja, Chontabamba and Matibamba were the areas of leishmaniasis higher transmission at Llaucano valley. The prevalence rate was 283,4/1000. Conclusions: Llaucano valley is an endemic Andean leishmaniasis area with fast spreading of this disease; disease transmission is associated to house factors and human behavior.

Key words: Leishmaniasis; risk factors; rural zones; poverty areas; Peru, Cajamarca, Llaucano valley.

\section{INTRODUCCIÓN}

En el Perú, la leishmaniasis tegumentaria constituye la segunda endemia de tipo tropical y la tercera causa de morbilidad por enfermedades transmisibles luego de la malaria y la tuberculosis, y cada año se presentan entre 7000 a 9000 nuevos casos probables. El área de riesgo representa el $74 \%$ del territorio nacional $\left({ }^{1}\right)$. La leishmaniasis es una de las enfermedades más antiguas en el Perú, y durante siglos se ha mantenido endémica en comunidades nativas de las vertientes occidentales y valles interandinos, llegando a constituirse en una enfermedad semidoméstica $\left({ }^{2}\right)$. Desde los primeros estudios acerca de la leishmaniasis, se sabe que son dos las formas principales de esta enfermedad que tienen importancia clínica y epidemiológica: la leishmaniasis cutánea andina y la leishmaniasis selvática, cada una con patrones clínico-epidemiológicos propios.

La leishmaniasis andina cutánea «uta» es endémica en las vertientes occidentales del norte y centro de la cordillera de los Andes y en ciertos valles interandinos, desde los $5^{0}$ hasta los $15^{\circ}$ de latitud sur y entre 1100 a $3000 \mathrm{msnm}\left({ }^{3,4}\right)$. La "uta" se transmite activamente independiente del sexo y afecta con mayor frecuencia a los niños en edad escolar y preescolar de las áreas rurales o periurbanas, donde esta enfermedad es endémica $\left({ }^{5}\right)$. La alta incidencia de la leishmaniasis a temprana edad, con lesiones mayormente ubicadas en cara y miembros superiores, estaría en relación a una mayor exposición domiciliaria a los vectores, por lo siguiente: a) ubicación de las viviendas en las zonas donde se distribuyen los vectores, b) desplazamiento de las familias de las zonas altas hacia las áreas de cultivo en el fondo de los valles, o c) el pastoreo de ganado, que generalmente es realizado por niños y adolescentes en focos de transmisión de leishmaniasis $\left({ }^{6-11}\right)$. Las lesiones cutáneas puras son predominantes y responden favorablemente al tratamiento con antimoniales o curan espontáneamente, mientras que las lesiones mucosas se dan generalmente por contigüidad a partir de una lesión cutánea adyacente, o en casos raros por metástasis $\left({ }^{10}\right)$.

La leishmaniasis selvática es endémica en valles orientales y en toda la llanura amazónica, por debajo de los $1800 \mathrm{msnm}\left({ }^{12}\right)$. La leishmaniasis selvática es una enfermedad típicamente zoonótica, en la cual la infección humana es accidental, cuando el hombre se interna en el bosque donde se mantiene el ciclo natural de la leishmaniasis entre el parásito, los vectores y reservorios silvestres, para realizar actividades agrícolas, extracción de oro, explotación minera y petrolera, investigación científica, maniobras militares, etc. $\left({ }^{12,13}\right)$. La leishmaniasis selvática afecta principalmente a los adultos del sexo masculino entre 20 a 40 años de edad, y las lesiones cutáneas se presentan con mayor frecuencia en miembros inferiores, lo que estaría en relación a un mayor contacto hombre-vector en áreas extradomiciliarias $\left({ }^{14}\right)$. En un número indeterminado de casos, la leishmaniasis selvática es de carácter progresivo, presentando dos fases: una cutánea pura, similar a la leishmaniasis cutánea andina, y otra donde se produce metástasis que compromete a la mucosa nasoorofaríngea. Las lesiones mucosas, cuando se presentan, por lo general son rebeldes al tratamiento convencional. Estas últimas pueden aparecer meses o años después de haber cicatrizado la lesión cutánea inicial $\left({ }^{12}\right)$.

En los estudios epidemiológicos sobre la leishmaniasis tegumentaria en nuestro país, varios factores han sido asociados con el riesgo 
de transmisión de esta enfermedad. Entre estos, la actividad ocupacional es la más extensamente relacionada con la leishmaniasis selvática $\left({ }^{12}\right)$. Llanos-Cuentas $\left({ }^{3}\right)$, en un estudio tipo casocontrol en 5 áreas endémicas de leishmaniasis cutánea andina de los departamentos de Lima, Ancash y Piura, identificó como factores de riesgo la ubicación, el tipo de construcción y las características ecológicas alrededor de la vivienda; el hacinamiento en que viven las personas y algunas actividades realizadas por los pobladores. Estos factores implican que la transmisión de la "uta" ocurre en a) en el interior de las casas, b) alrededor de la vivienda, c) en el ambiente doméstico, pero no claramente definido si es dentro o fuera de las casas, y d) en áreas extradomiciliarias.

No se tiene referencias de estudios acerca de la leishmaniasis tegumentaria en la provincia de Chota; y son pocos los trabajos epidemiológicos realizados en otras zonas del departamento de Cajamarca. En el Censo de población de 1940, donde se recogió datos sobre diversas patologías a nivel nacional, se menciona la existencia de casos de "uta" en esta provincia $\left({ }^{5}\right)$. Medina $\left({ }^{15}\right)$, en su "Monografía de Chota" menciona: “... La uta, se presenta en Querocoto, Paccha y Pión”.

El valle de Llaucano, materia de este estudio, está ubicado en la provincia de Chota, Cajamarca. En algunas localidades del valle, un número considerable de personas, en su mayoría niños, está afectado por la leishmaniasis tegumentaria. Los casos se han presentado en forma esporádica durante varias décadas; sin embargo, en la última década se ha observado un comportamiento epidémico de la enfermedad, lo que ha motivado el desarrollo de este estudio, para lo cual se plantearon los siguientes objetivos:

1. Determinar la presencia de casos de leishmaniasis en la población del valle de Llaucano y su prevalencia.

2. Determinar los factores que determinan la transmisión de la leishmaniasis tegumentaria en el valle de Llaucano.

\section{MATERIAL Y MÉTODOS}

El área de estudio comprendió ocho localidades de los distritos de Paccha y Chadín, provincia de Chota (Cajamarca), ubicadas a ambas márgenes del río Llaucano, que recorre de sur a norte la provincia de Hualgayoc, para luego ingresar a la provincia de Chota atravesando los distritos de Paccha, Chadín y Pión y desembocar finalmente en el río Marañón (Figuras 1 y 2). Las localidades estudiadas están ubicadas entre los 1720 hasta los $2500 \mathrm{msnm}$.

El valle de Llaucano presenta clima tropical con temperatura media anual superior a $25^{\circ} \mathrm{C}$; mientras que en las alturas, el clima es templado y frío (la temperatura media anual promedio es de $\left.12^{\circ} \mathrm{C}\right)$. La precipitación pluvial anual es superior a los $1000 \mathrm{~mm}$, siendo más abundante durante los meses de enero a marzo. La humedad relativa oscila entre 60 y $98 \%$. El valle es habitado por comunidades campesinas que tienen como actividad principal la agricultura complementada con la crianza de animales domésticos, y en menor escala el comercio. Los cultivos agrícolas son estacionales de acuerdo al régimen de lluvias, y en el trabajo de campo generalmente participan todos los miembros de la familia, incluyendo a los niños. Las viviendas son rústicas en la zona rural, con paredes de "tapia" (mezcla de barro y piedra) y piso de tierra, mientras que en el área urbana las condiciones mejoran (paredes de adobe revestido con yeso y piso de madera o cemento).

Entre agosto de 1994 y julio de 1998, se realizó entrevista personal casa por casa entre la población del valle de Llaucano expuesta a la leishmaniasis tegumentaria, lo que permitió obtener información que incluyó: nombre, edad, sexo, ocupación, grado de instrucción, tiempo de residencia en el área, viajes a otras zonas. Historia de cicatrices o lesiones cutáneas no dolorosas de más de 30 días de duración, localización y número de lesiones; fecha, lugar, circunstancia en que adquirió la enfermedad y tiempo de duración de ésta; tratamiento recibido, 


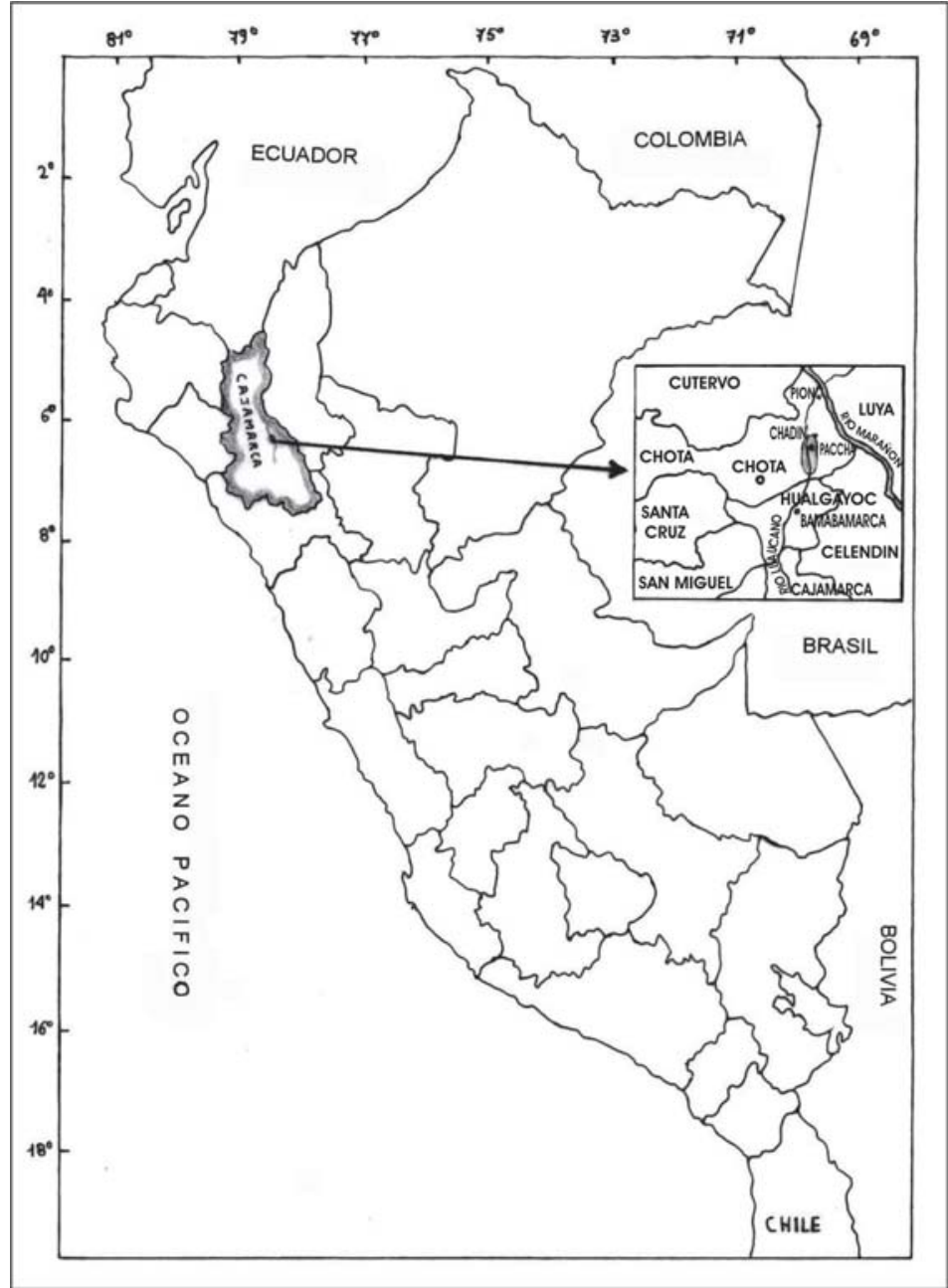

Figura 1. Ubicación del área de estudio en el territorio nacional.

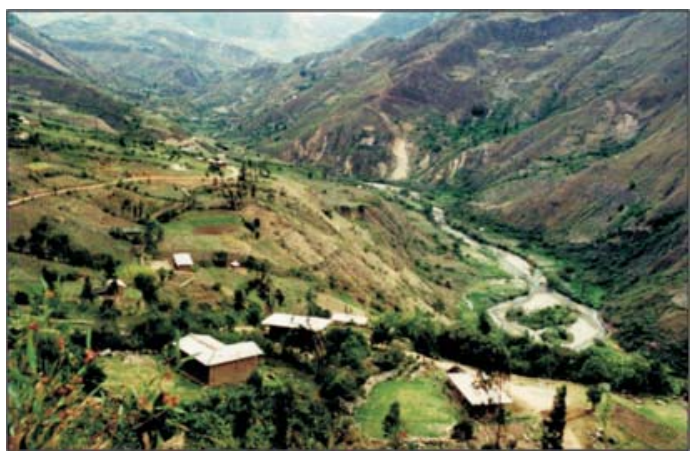

Figura 2. Vista panorámica del valle Llaucano. exámenes de laboratorio efectuados e información complementaria si la hubiera. Así mismo se obtuvo información concerniente a datos de vivienda, como altitud, material de construcción, cultivos principales, animales domésticos y silvestres, protección antivectorial. La información obtenida se registró en fichas epidemiológicas y clínicas.

El estudio clínico de casos de leishmaniasis fue realizado por un médico especialista en enfermedades tropicales. Se consideró como casos positivos aquellos que se ajustaban a alguno de los siguientes parámetros:

a) Lesiones cutáneas localizadas en partes expuestas del cuerpo, clínicamente compatibles con leishmaniasis tegumentaria y antecedentes epidemiológicos.

b) Aislamiento del parásito a partir de las lesiones cutáneas y/o cutaneomucosas activas.

c) Visualización microscópica de amastigotes de Leishmania en los frotises o biopsias.

d) Signos clínicos compatibles con leishmaniasis más intradermorreacción de Montenegro positiva.

Los datos obtenidos fueron registrados en una tabla de datos en Microsoft Visual Fox Pro. Las variables fueron analizadas mediante un programa de ordenador para la investigación en Ciencias de la Salud, Epi Info 2000. La identificación de posibles factores de riesgo asociados con la transmisión de la leishmaniasis, se realizó mediante el cálculo del odds ratio (OR) con intervalos de confianza (i.c.) de Cornfield al $95 \%$, y el valor de $p\left({ }^{16,17}\right)$. Para determinar el OR, se definió dos grupos de personas: un grupo con diagnóstico positivo de leishmaniasis (casos) y otro grupo sin la enfermedad (no casos 
o grupo control). Se comparó ambos grupos con los probables factores de exposición a la leishmaniasis, mediante la utilización de tablas 2x2 en el programa Epi Info 2000.

Para los fines de este estudio, se consideró un valor de $\mathrm{OR}>1,5$ como indicador de una asociación evidente entre el factor de riesgo y la leishmaniasis. Un intervalo de confianza mayor de 1,5, indica una asociación entre el factor de riesgo y la enfermedad; mientras que los intervalos que contienen a 1 ó son menores que 1,5 , indican que no hay relación evidente entre el factor de riesgo y la enfermedad $\left({ }^{18,19}\right)$.

\section{RESULTADOS}

Se encuestó 727 personas, $349(48,0 \%)$ hombres y $378(51,9 \%)$ mujeres distribuidos en 140 viviendas, lo que representa aproximadamente el $20 \%$ de la población del valle. La edad promedio de la población estudiada fue de 22,7 años, con un rango que varió entre menores de 1 año hasta 85 años.

Se diagnosticó $206(28,3 \%)$ casos de leishmaniasis tegumentaria de un total de 727 personas encuestadas, $143(69,4 \%)$ con cicatrices y $63(30,6 \%)$ con lesiones activas; 204 casos de leishmaniasis cutánea y 2 cutáneomucosa, siendo las localidades de Matibamba, Chontabamba y Paccha Baja las más afectadas. Del total de casos, $111(53,9 \%)$ correspondieron al sexo masculino y $95(46,1 \%)$ al femenino. El grupo comprendido entre 5 y 9 años de edad es el que presentó la más alta prevalencia $(40,6 \%)$ (Tabla 1$)$. La edad promedio de la población afectada por la leishmaniasis tegumentaria fue de 22,4 años (21,2 años en el sexo masculino y 23,8 en el sexo femenino), con una mediana de 14 años para ambos sexos. El $31,1 \%$ de las lesiones estuvo localizada en miembros superiores; $28,6 \%$ en cara; y $30,6 \%$, en miembros inferiores. El 3,4\% presentó lesiones en pabellón auricular y $6,3 \%$; lesiones múltiples ubicadas en diferentes regiones del cuerpo (Figura 3).
Tabla 1. Población estudiada por grupos de edad, casos de leishmaniasis y análisis epidemiológico de la edad como factor de riesgo.

\begin{tabular}{lrrrccr}
\hline $\begin{array}{l}\text { Grupos } \\
\text { de edad }\end{array}$ & PE & $\begin{array}{r}\text { LT } \\
(+)\end{array}$ & $\begin{array}{r}\text { LT } \\
(-)\end{array}$ & OR & IC95\% & $p$ \\
\hline $0-4$ & 89 & 12 & 77 & 0,33 & $0,13-0,87$ & 0,01245 \\
$5-9$ & 138 & 56 & 82 & 1,46 & $0,67-3,20$ & 0,029957 \\
$10-14$ & 117 & 38 & 79 & 1,03 & $0,46-2,32$ & 0,93654 \\
$15-19$ & 73 & 18 & 55 & 0,70 & $0,28-1,74$ & 0,40199 \\
$20-39$ & 161 & 40 & 121 & 0,71 & $0,32-1,56$ & 0,35322 \\
$40-59$ & 105 & 28 & 77 & 0,78 & $0,34-1,80$ & 0,52515 \\
$>60$ & 44 & 14 & 30 & 1,28 & $0,55-2,95$ & 0,52515 \\
Total & 727 & 206 & 521 & & & \\
\hline
\end{tabular}

PE: Población estudiada OR: Odds Ratio

LT: Casos de leishmaniasis tegumentaria

IC: Intervalos de confianza

Todos los grupos de edad estuvieron afectados por la leishmaniasis tegumentaria en el valle de Llaucano. No existió relación entre el sexo y el riesgo para contraer la leishmaniasis: OR 1,39, CI95\% 0,99-1,95 para el sexo masculino; y OR 0,72, CI95\% 0,51-1,01 para el femenino.

La actividad ocupacional habitual no fue un factor de riesgo para contraer la leishmaniasis tegumentaria en el valle de Llaucano (CI95\% $<1,50$ ), a pesar de que los agricultores y los estudiantes fueron los más expuestos (Tabla 2).

Las personas que duermen en viviendas temporales ubicadas en medio de las chacras durante la cosecha de café, principalmente,

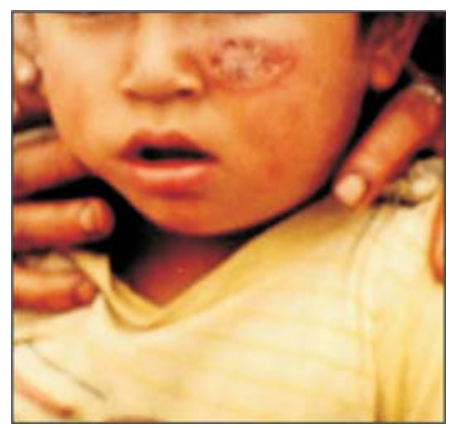

Figura 3. Lesión ulcerativa cutánea en un caso de leishmaniasis del valle Llaucano 
Tabla 2. Factores de exposición a la leishmaniasis en relación a la ocupación y la actividad desarrollada al momento de contraer la enfermedad.

\begin{tabular}{|c|c|c|c|c|c|}
\hline $\begin{array}{l}\text { LT } \\
(+)\end{array}$ & $\begin{array}{l}\text { LT } \\
(-)\end{array}$ & Total & OR & IC95\% & $p$ \\
\hline
\end{tabular}

Ocupación actual

$\begin{array}{lrrrrrr}\text { Agricultores } & 106 & 198 & 304 & 1,73 & 1,25-2,39 & 0,00092 \\ \text { Amas de casa } & 72 & 203 & 275 & 0,84 & 0,60-1,18 & 0,31513 \\ \text { Estudiantes } & 88 & 151 & 239 & 1,83 & 1,31-2,56 & 0,00039 \\ \text { Preescolares } & 19 & 72 & 91 & 0,45 & 0,27-0,74 & 0,06538 \\ \text { Otros } & 6 & 37 & 43 & 0,39 & 0,16-0,94 & 0,03107\end{array}$

Actividad al momento de contraer la enfermedad

$\begin{array}{lrrrrrr}\text { Recoger leña } & 100 & 143 & 243 & 2,49 & 1,79-3,48 & 0,00001 \\ \text { Recoger agua } & 92 & 161 & 253 & 1,80 & 1,30-2,51 & 0,00045 \\ \begin{array}{l}\text { Lavar ropa } \\ \text { Hilar/tejer }\end{array} & 47 & 159 & 206 & 0,67 & 0,46-0,98 & 0,03795 \\ \begin{array}{l}\text { Trabajo en } \\ \text { chacra }\end{array} & 6 & 8 & 0,84 & 0,17-4,20 & 0,83337 \\ \begin{array}{l}\text { Dormir en vivienda } \\ \text { temporal }\end{array} & 149 & 189 & 338 & 4,59 & 3,22-6,54 & 0,00000 \\ \begin{array}{l}\text { Descansando en } \\ \text { peridomicilio } 178\end{array} & 414 & 592 & 2 & 1,05-2,58 & 0,03011\end{array}$

LT: Casos de leishmaniasis tegumentaria OR: Odds ratio IC: Intervalos de confianza

estuvieron más expuestas a contraer la leishmaniasis (OR 4,59, CI95\% 3,22-6,54). Recoger leña (OR 2,49, CI95\% 1,79-3,48) en los matorrales circundantes a las viviendas o plantaciones de árboles, actividad que generalmente es realizada por los menores de edad y las amas de casa, también constituyó un factor de riesgo para contraer la enfermedad: OR 2,49, CI95\% 1,79-3,48 (Tabla 2).

Las viviendas dispersas ubicadas en áreas rurales (Tabla 3) estuvieron expuestas a la transmisión de la leishmaniasis (OR 3,97, IC95\% $1,94-8,14)$, mientras que las que se encuentran en áreas urbanas o formando conglomerados están protegidas (IC95\% límite inferior <1,0). Las viviendas que tienen 6 ó más residentes viviendo en estado de hacinamiento están más expuestas a la enfermedad, que aquellas donde viven cinco o
Tabla 3. La vivienda como factor de riesgo para contraer la leishmaniasis $(n=140)$.

\begin{tabular}{|c|c|c|c|c|c|c|}
\hline Variable & $\begin{array}{l}\mathrm{LT} \\
(+)\end{array}$ & $\begin{array}{l}\mathrm{LT} \\
(-)\end{array}$ & Total & OR & IC95\% & $p$ \\
\hline \multicolumn{7}{|c|}{ Ubicación viviendas } \\
\hline Disperso & 59 & 26 & 85 & 3,97 & $1,94-8,14$ & 0,00013 \\
\hline Conglomerado & 12 & 7 & 19 & 1,38 & $0,51-3,75$ & 0,52606 \\
\hline Área urbana & 8 & 28 & 36 & 0,13 & $0,05-0,32$ & 0,00001 \\
\hline \multicolumn{7}{|c|}{ Número de residentes por vivienda } \\
\hline $2-5$ & 35 & 44 & 79 & 0,31 & $0,14-0,66$ & 0,00104 \\
\hline$>6$ & 4 & 17 & 61 & 325 & $150-710$ & 0,00104 \\
\hline
\end{tabular}

Número de ambientes por vivienda

$\begin{array}{lllllll}2 & 48 & 16 & 64 & 4,35 & 1,98-9,66 & 0,00001 \\ >3 & 31 & 45 & 76 & 0,23 & 0,10-0,50 & 0,00001\end{array}$

Piso viviendas

$\begin{array}{lllllll}\text { Tierra } & 66 & 33 & 99 & 4,31 & 1,98-9,39 & 0,00016\end{array}$

Tabla/cemento $13 \quad 28 \quad 41 \quad 0,23 \quad 0,10-0,54 \quad 0,00016$

Pared viviendas

$\begin{array}{lllllll}\text { Tapial } & 62 & 33 & 95 & 3,09 & 1,50-6,46 & 0,00227 \\ \text { Adobe } & 13 & 32 & 45 & 0,18 & 0,08-0,39 & 0,00001\end{array}$

Tipo de vegetación alrededor de la vivienda

$\begin{array}{lrrrrrr}\text { Café } & 66 & 24 & 90 & 7,83 & 3,57-17,17 & 0,00000 \\ \text { Caña de azúcar } & 56 & 20 & 76 & 4,99 & 2,42-10,27 & 0,00001 \\ \text { Frutas } & 41 & 14 & 55 & 3,62 & 1,72-7,61 & 0,00053 \\ \text { Maíz } & 48 & 24 & 72 & 2,39 & 1,20-4,73 & 0,01225 \\ \text { Tubérculos } & 33 & 14 & 47 & 2,41 & 1,14-5,08 & 0,01981\end{array}$

Presencia de animales en el medio domiciliario

Intradomicilio $\quad 63 \quad 44 \quad 107 \quad 1,52 \quad 0,69-3,33 \quad 0,29421$

$\begin{array}{lllllll}\text { Peridomicilio } & 68 & 37 & 105 & 4,01 & 1,77-9,09 & 0,00061\end{array}$

$\begin{array}{lllllll}\text {-aves } & 26 & 22 & 48 & 0,87 & 0,43-1,76 & 0,69767\end{array}$

$\begin{array}{lllllll}\text {-perros } & 51 & 19 & 70 & 4,03 & 1,98-8,20 & 0,00009\end{array}$

-ganado vacuno $20 \quad 14 \quad 34 \quad 1,14 \quad 0,52-2,54 \quad 0,74707$

$\begin{array}{llllllll}\text {-equinos } & 12 & 6 & 18 & 1,64 & 0,58-4,66 & 0,34977\end{array}$

$\begin{array}{lllllll}\text {-roedores } & 65 & 43 & 108 & 1,94 & 0,88-4,32 & 0,10081\end{array}$

-marsupiales $\quad 47 \quad 20 \quad 67 \quad 3,01 \quad 1,50-6,05 \quad 0,00178$

Flebotominos (Lutzomyia spp.)

“manta blanca” $62 \quad 24 \quad 86 \quad 5,62 \quad 2,68-11,82 \quad 0,00001$

LT: Casos de leishmaniasis tegumentaria

OR: Odds ratio IC: Intervalos de confianza

menos personas (OR 3,25, IC95\% 1,50-7,10). El tipo de construcción de las viviendas como factor de riesgo está en relación con la ubicación: las viviendas de las áreas rurales tienen piso de tierra (OR 4,31, IC95\% 1,98-9,39) y paredes de 
Figura. 4. Leishmaniasis tegumentaria en el valle de Llaucano focos de transmision de la leishmaniasis y ubicación de las viviendas.

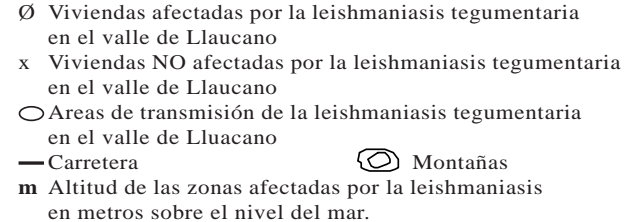

"tapia" (OR=3,09, IC95\% 1,48-6,46), por lo que están más expuestas a la transmisión de la leishmaniasis, mientras que las viviendas ubicadas en áreas urbanas, con paredes de adobe $(\mathrm{OR}=0,18$, IC95\%0,08$0,39)$ y pisos de madera y/o cemento $(\mathrm{OR}=0,23, \mathrm{IC} 95 \%$ 0,10-0,54) estuvieron mejor protegidas. La presencia de café $(\mathrm{OR}=7,83$, IC95\% 3,57-17,17), caña de azúcar (OR 4,99, IC95\% 2,42-10,27) y frutas (OR 3,62, IC95\% 1,72-7,61) en los alrededores de las viviendas, expuso a sus moradores a contraer la leishmaniasis tegumentaria. Existió una correlación lineal positiva entre el número de residentes por vivienda y el número de afectados por la leishmaniasis dentro de la misma $\left(r=0,754, r^{2}=0,569\right)$.

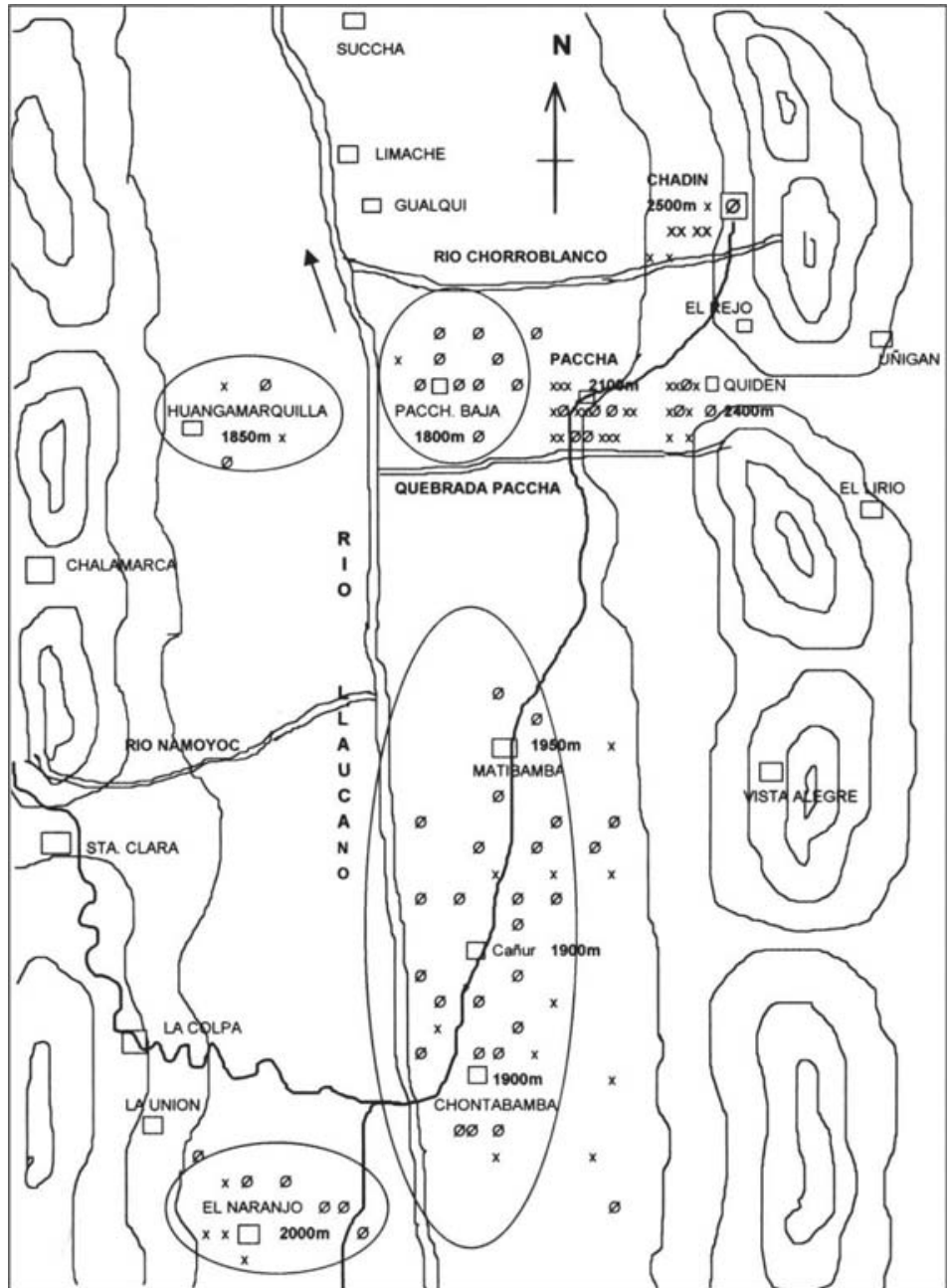

Tabla 4. Prevalencia acumulada de leishmaniasis (lesiones activas y cicatrices) por grupos de edad, en la población estudiada.

\begin{tabular}{|c|c|c|c|c|c|c|c|c|c|}
\hline \multirow{3}{*}{$\begin{array}{l}\text { Grupos } \\
\text { de edad }\end{array}$} & \multicolumn{6}{|c|}{ Sexo } & \multicolumn{3}{|c|}{ Total } \\
\hline & \multicolumn{3}{|c|}{ Hombres } & \multicolumn{3}{|c|}{ Mujeres } & \multirow[b]{2}{*}{$\mathrm{PE}$} & \multirow[b]{2}{*}{$\mathrm{LT}(+)$} & \multirow[b]{2}{*}{$\% \mathrm{LT}(+)$} \\
\hline & $\mathrm{PE}$ & $\mathrm{LT}(+)$ & $\% \mathrm{LT}(+)$ & $\mathrm{PE}$ & $\mathrm{LT}(+)$ & $\% \mathrm{LT}(+)$ & & & \\
\hline $0-4$ & 41 & 7 & 17,2 & 48 & 5 & 10,4 & 89 & 12 & 13,5 \\
\hline $5-9$ & 71 & 29 & 40,9 & 67 & 27 & 40,3 & 138 & 56 & 40,6 \\
\hline $10-14$ & 65 & 22 & 33,9 & 52 & 16 & 30,8 & 117 & 38 & 32,5 \\
\hline $15-19$ & 33 & 12 & 36,4 & 40 & 6 & 15,0 & 73 & 18 & 24,7 \\
\hline $20-39$ & 74 & 19 & 25,7 & 87 & 21 & 24,1 & 161 & 40 & 24,8 \\
\hline $40-59$ & 53 & 18 & 33,9 & 52 & 10 & 19,2 & 105 & 28 & 26,8 \\
\hline $60-+$ & 12 & 4 & 33,3 & 32 & 10 & 31,3 & 44 & 14 & 31,8 \\
\hline Total & 349 & 111 & 31,8 & 378 & 95 & 25,1 & 727 & 206 & 28,3 \\
\hline
\end{tabular}

PE: Población estudiada LT: Casos de leishmaniasis tegumentaria

$\%$ LT: Tasa de prevalencia por grupos de edad

Tasa de prevalencia acumulada : $\quad T=283,4 / 1000 h a b$ 
La presencia de animales en el peridomicilio constituyó un factor de riesgo para la leishmaniasis (OR=4,01, IC95\% 1,77-9,09). Entre los animales domésticos, las viviendas que tenían perros estuvieron más expuestas que aquellas donde existió otro tipo de animales (OR 4,03 , IC95\% 1,98-8,20). Los animales silvestres, principalmente los marsupiales (Didelphis spp. "muca") merodean las viviendas en busca de alimento (OR 3,01, IC95\% 1,50$6,05)$ y los flebotominos ingresan a éstas a través de los agujeros de las paredes de "tapia". La presencia de "manta blanca" (Lut-zom-yia) en el interior de los domicilios estuvo asociada a una fuerte exposición domiciliaria a la enfermedad (OR 5,62, IC95\% 2,68-11,82).

El 27,2\% de personas adquirió la leishmaniasis en Chontabamba, 25,2\%, en Matibamba y $30,6 \%$, en Paccha Baja. Estas tres localidades constituyen los focos de más alta transmisión de la enfermedad. Según los datos obtenidos en este estudio, los casos de leishmaniasis observados en Paccha (2 $100 \mathrm{msnm}$ ), Quidén (2 $400 \mathrm{msnm}$ ) y Chadín (2 $500 \mathrm{msnm}$ ) han sido adquiridos en Paccha Baja, Huangamarquilla, Matibamba y Chontabamba (todas ubicadas por debajo de los $2100 \mathrm{msnm}$ ), hacia donde migra la mayoría de los pobladores para realizar sus actividades agrícolas (Figura 4).

La tasa de prevalencia acumulada de leishmaniasis en la población estudiada fue $31,8 \%$ en el sexo masculino y de $25,1 \%$ en el femenino. El grupo de 5 a 9 años de edad presentó la más alta tasa de prevalencia $(40,6 \%)$ seguido de los de 10 a 14 años $(32,5 \%)$ y los mayores de $60(31,8 \%)$, mientras que el grupo de 0 a 4 años presentó la tasa más baja $(13,5 \%)$. La tasa de prevalencia acumulada de leishmaniasis tegumentaria en la población estudiada fue 283,4/1 000 hab (Tabla 4).

\section{DISCUSIÓN}

El valle de Llaucano es un valle interandino que presenta caracteçrísticas ecológicas de selva alta que favorecen la presencia de los elementos que forman la cadena epidemiológica de la leishmaniasis: casos humanos con lesiones en las que se ha determinado la presencia de amastigotes de Leishmania; en los casos de lesiones cicatrizales, con Intradermorreacción de Montenegro positiva, animales silvestres y domésticos probables reservorios del parásito; y flebotominos vectores potenciales de la enfermedad, como sucede en otras áreas endémicas de leishmaniasis tegumentaria.

La ocurrencia de infección en ambos sexos, más frecuente en niños que en adultos, con lesiones mayormente ubicadas en cara y miembros superiores, sugiere una transmisión intradomiciliaria de la leishmaniasis en el valle de Llaucano, ya que hombres y mujeres están expuestos por igual a la picadura de los flebotominos. Observaciones similares han sido realizadas por Cordero $\left({ }^{20}\right)$ en Santa Cruz, Ancash; Butrón $\left({ }^{21}\right)$ en Surco, Lima; Miranda $\left.{ }^{8}\right)$ en La Libertad, quienes resaltan esta característica epidemiológica de la leishmaniasis cutánea andina. Las lesiones ubicadas en miembros inferiores, principalmente en mujeres, se deberían al resultado de picaduras en medios peridomésticos o en campo abierto, y estaría en relación a las labores agrícolas y el trabajo doméstico, característica observada por Barrera ( ${ }^{11}$ ) en focos endémicos de leishmaniasis andina del Ecuador.

Las viviendas ubicadas en las áreas rurales del valle de Llaucano están expuestas a la leishmaniasis, mientras que las que se encuentran en áreas urbanas están protegidas. Seis o más personas durmiendo en la misma habitación en estado de hacinamiento, con animales domésticos dentro de la vivienda, constituye un factor de riesgo, pues atraen a los flebotominos antropofílicos, los que ingresan libremente a través de los agujeros de las paredes de "tapia". Las plantaciones de café, caña de azúcar y frutas alrededor de las viviendas, son factores de riesgo que determinan un alto grado de exposición a la leishmaniasis tegumentaria (OR > 1,5; IC95\% 
$>1,5)$. Durante los periodos de actividad agrícola, los pobladores procedentes de las partes altas bajan al fondo del valle, donde permanecen varios días en viviendas improvisadas y duermen en condiciones precarias. Los terrenos de cultivo rodean a las viviendas, favoreciendo de esta manera la actividad hematófaga de los flebotominos en el medio domiciliario. Scorza $\left.{ }^{(22}\right)$ y Montoya $\left({ }^{23}\right)$, hacen énfasis en la superposición de las áreas cafetaleras y las de endemicidad de la leishmaniasis tegumentaria. La abundante vegetación alrededor de las viviendas y la adaptación del parásito a estos ambientes, permite el establecimiento de un ciclo domiciliario de la leishmaniasis $\left({ }^{14,24}\right)$.

Llanos-Cuentas $\left({ }^{3}\right)$, en áreas endémicas de leishmaniasis de los departamentos de Lima, Ancash y Piura, determinó como factores de riesgo para contraer esta enfermedad: vivir en casa con jardín (OR 2,8, IC95\% 1,1-4,1), tener ganado vacuno alrededor de la vivienda (OR 1,3, IC95\% 1,1-1,6) o tener vecinos con jardín en su casa (OR 2,9, IC95\% 1,3-6,9), debido a que los animales domésticos atraen a los flebotominos y las plantas constituyen una fuente de azúcares para éstos, favoreciendo su desarrollo; trabajar en irrigación de los terrenos de cultivo durante las noches por periodos de 8 a 12 horas (OR 2,2, IC95\% 1,2-4,2), pues las personas permanecen más tiempo expuestas a la picadura de los flebotominos.

El perro estaría jugando papel como reservorio secundario del parásito en el área de estudio, ya que su presencia en el medio domiciliario se asocia con la existencia de casos de leish-maniasis (OR $<1,5$, IC95\% $>1,5)$, como ha sido observado por Herrer $\left({ }^{25}\right)$ en el valle del Rímac, Aguilar $\left({ }^{26}\right)$ en Venezuela y Brasil, Mimori $\left({ }^{27}\right)$ y Dereure $\left({ }^{28}\right)$ en Ecuador. Este animal es susceptible de enfermarse al igual que el hombre, pero, a diferencia de éste, no siempre presenta lesión cutánea ulcerosa $\left({ }^{25}\right)$. Los roedores y marsupiales visitan ocasionalmente las viviendas en busca de alimento y podrían ser los reservorios silvestres del parásito en la zona, como se ha observado en otras áreas endémicas de leishmaniasis $\left({ }^{29}\right)$.

Las lesiones múltiples ubicadas en miembros superiores e inferiores son más graves que las lesiones únicas, pues producen un grado de incapacidad transitoria o permanente. El costo que demanda el tratamiento y el tiempo en que las personas permanecen inactivas a causa de la enfermedad pueden ser considerables. De esta manera, la leishmaniasis tegumentaria es una de las causas que dificulta el progreso de la región, pues afecta la economía de la población, provoca la disminución de la capacidad de trabajo de las personas afectadas y el temor entre los pobladores de las zonas vecinas.

Se concluye que, el valle de Llaucano, ChotaCajamarca, es una zona endémica de leishmaniasis tegumentaria, con una alta velocidad de propagación de la enfermedad, la que afecta con mayor frecuencia a los menores de 10 años. La transmisión de la leishmaniasis tegumentaria ocurre en ambientes intra y peridomiciliarios de las áreas rurales, siendo las localidades de Matibamba, Chontabamba y Paccha Baja las que presentan el mayor número de casos. Las personas que viven en estado de hacinamiento y en condiciones precarias en viviendas temporales o de "tapia” dispersas en el área rural rodeadas de café, frutas, caña de azúcar y con animales domésticos en el peridomicilio, están más expuestas a contraer la enfermedad, mientras que en el área urbana la población está protegida.

\section{REFERENCIAS BIBLIOGRÁFICAS}

1. Ministerio de Salud. Doctrina, normas y procedimien-tos para el control de las leishmaniasis en el Perú. Lima: Ministerio de Salud; 1995. p. 65.

2. Herrer A, Hidalgo V, Meneses O. Leishmanasis tegumentaria e insecticidas en el Perú. Reactivación de la uta durante los últimos años. Rev Inst Med Trop Sao Paulo. 1980;22(4):203-6.

3. Llanos-Cuentas A. Risk factors associated with the transmission of andean cutaneous leishmaniasis. Thesis submitted for the degree of Doctor of Philosophy in the University of London. 1993. p. 162. 
4. Villaseca P, Llanos-Cuentas A, Pérez E, Davies C. A comparative field study of the relative importance of Lutzomyia peruensis and Lutzomyia verrucarum as vectors of cutaneus leishmaniasis in the peruvian Andes. Am J Trop Med and Hyg. 1993;49(2):260-9.

5. Weiss P. Epidemiología y clínica de las leishmaniosis tegumentarias en el Perú. Rev Med Exp. 1943;2(3):209-48.

6. Weiss P. Las zonas andinas de patología de Phlebotomus. An Fac Med Lima. 1953;36(1):1-11.

7. Herrer A. Verruga y uta en el valle de Huaillacayán. Departamento de Ancash. Rev Med Exp. 1957;11(1-2):40-9.

8. Miranda H, Cruzado L, Ortiz S. Leishmaniasis cutánea andina: aspectos clínicos y etiológicos. Libro de Resúmenes V Congreso Panamericano de Infectología, Lima. 1991. p. 17.

9. Cáceres A, Villaseca P, Inga R, López M, Arévalo J, Llanos-Cuentas A. Lutzomyia ayacuchensis (Diptera: Psychodidae, Phlebotominae) probable vector de la leishmaniasis cutánea andina (uta) en Lucanas y Parinacochas (Ayacucho, Perú). Libro de Resúmenes V Congre-so Panamericano de Infectología, Lima. 1991. p.22.

10. Soria J. Leishmaniasis tegumentaria, casos diagnosticados en el Instituto de Medicina Tropical, 1975-1990. Rev Per Med Trop UNMSM. 1993;7(2):59-80.

11. Barrera C, Herrera M, Martínez F, León R, Richard A, Guderian R, et al. Leishmaniose en Equateur. 1. Incidence de la leishmaniose tegumentaire sur la falcade Pacifique. Ann Soc Belge Med Trop. 1994;74:1-12.

12. Tejada A. Leishmaniasis tegumentaria en el Perú. Investigación epidemiológico-clínica de la leishmaniasis tegumentaria en los departamentos del Cuzco y Madre de Dios. Tesis para optar el grado de Doctor en Medicina. UNMSM. Lima. 1973. p. 210.

13. Lainson R, Shaw J, Silveira F, De Souza A, Braga R, Ishikawa E. The dermal leishmaniases of Brazil, with special reference to the eco-epidemiology of the disease in amazonía. Mem Inst Oswaldo Cruz, Río de Janeiro. 1994;89(3):435-43.

14. Castro Gomes A. Sand fly vectorial ecology in the state of Sao Paulo. Mem Inst Oswaldo Cruz, Río de Janei-ro. 1994;89(3):457-60.

15. Medina A. Monografía de Chota. Lima: T. Schouch S.A.; 1962.

16. Carpenter TE. Epidemiologic programs for computers and calculators. Decision- tree analysis using a microcomputer. Am J Epidem. 1989;124(5):843-50.

17. Gardner M, Altman D. Intervalos de confianza y no valores p: estimación en vez de pruebas de hipótesis. Bol Of Sanit Panam. 1993;114(6):536-49.

18. Rosner B, Hennekens CH. Analytic methods in matched pair epidemiologic studies. Int J Epidem. 1978;(7):367-72.

19. Baltazar J. El potencial del método de casos y controles para las evaluaciones epidemiológicas rápidas. Bol Of Sanit Panam. 1994;117(1):44-52.
20. Cordero F, Zevallos J, Sihuincha M. Aspectos epidemiológicos de la leishmaniasis tegumentaria americana en el distrito de Santa Cruz, Ancash. Diagnóstico. 1991;28(1/2):11-4.

21. Butrón B. Leishmaniasis cutánea andina en el distrito de Surco. Tesis Bachiller en Medicina. UPCH. 1982.

22. Scorza J, Rojas, E. Caficultura y leishmaniasis tegumentaria en Venezuela. Bol Dir Malariol Saneam Ambient. 1988;28(3/4):114-27.

23. Montoya J, Jaramillo C, Palma G, Gomez T, Segura J, Travi, B. Report of an epidemic outbreak of tegumentary leishmaniasis in a coffee-growing area of Colombia. Mem Inst Oswaldo Cruz, Río de Janeiro. 1990;85(1):119-21.

24. Góm ez E, Hashiguchi Y. An ecological view of leishmaniasis-endemic areas in Ecuador. Studies on the World Leishmaniasis and its Transmission, with Particular Reference to Ecuador. Kochi City, Japan: Kiowa Printing \& Co. Ltd. 1987. p. 174

25. Herrer A. Repercusión del uso casero de los insecticidas en la incidencia de la leishmaniasis tegumentaria del perro. Rev Med Exp. Lima. 1956;10(2):139-44.

26. Aguilar C, Rangel E, García I, Fernández E, Momen H, Grimaldi G, et al. Zoonotic cutaneous leishmaniasis due to Leishmania (Viannia) braziliensis associated with domestic animals in Venezuela and Brazil. Mem Inst Oswaldo Cruz. 1989;84(1):19 28.

27. Mimori T, Sud R, Gómez E, Hashiguchi Y. A seroepidemiological survey of canines in a area endemic for andean leishmaniasis in Ecuador. Studies on the World Leishmaniasis and its Transmission, with Particular Reference to Ecuador. Kochi City, Japan: Kiowa Printing \& Co. Ltd. 1992. p. 182.

28. Dereure J, Espinel I, Barrera C, Guerrini F, Martín A, Echevarría R, et al. Leishmaniose en Equateur. 4. Infestation naturelle du chien par Leishmania panamensis. Ann Soc Belge Med Trop. 1994;74:29-33.

29. Gomes A, Coutinho S, Paim G, Oliveira S, Galati E, Nunes $\mathrm{M}$, et al. Aspectos ecológicos da leishmaniose tegumentar americana. 8. Avaliacao da atividade enzoótica de Leishmania (Viannia) braziliensis, em ambiente florestal e peridomiciliar, regiao do Vale do Ribeira, Estado de Sao Paulo, Brasil. Rev Inst Med Trop Sao Paulo. 1990;32(2):105-15.

Manuscrito recibido el 08 de febrero de 2005 y aceptado para publicación el 15 marzo 2005.

\section{Correspondencia: Blgo. Víctor Zorrilla}

Instituto de Medicina Tropical "Daniel A. Carrión", UNMSM

José Santos Chocano 199. Bellavista, Callao

Correo-e: victor_zorrilla@hotmail.com

victorzorrillac@yahoo.es 